\title{
Relación entre el factor de crecimiento hepático y el estadio de la cirrosis
}

\section{Relationship between Hepatocyte Growth Factor (Hgf) and Stage of Cirrosis}

Ismael de Jesús Yepes Barreto, PhD, ${ }^{1}$ María Nicol Múnera Contreras, MD, ${ }^{2}$ Amileth Suárez-Causado, PhD. ${ }^{3}$

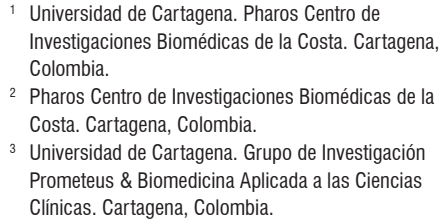

2 Pharos Centro de Investigaciones Biomédicas de la Costa. Cartagena, Colombia.

3 Universidad de Cartagena. Grupo de Investigación Prometeus \& Biomedicina Aplicada a las Ciencias Clínicas. Cartagena, Colombia.

\begin{abstract}
Resumen
Introducción: en el hígado, el factor de crecimiento hepático $(\mathrm{FCH})$ es conocido por ser un potente agente mitogénico tanto in vivo como in vitro. Sin embargo, el papel del $\mathrm{FCH}$ en la cirrosis no está completamente claro y algunos estudios lo señalan como un marcador de severidad en la cirrosis, en la insuficiencia hepática aguda y en la hepatitis crónica. Objetivos: determinar la relación entre el FCH y el estadio de la cirrosis hepática e identificar los factores asociados con los niveles de FCH en esta población. Metodología: se evaluaron todos los pacientes con cirrosis hepática atendidos desde enero a marzo de 2014. La elastografía transitoria (ET), la recopilación de la información clínica y la extracción de la muestra para la determinación del FCH se realizó de forma simultánea en el momento de la inclusión. Resultados: no se encontró relación entre los niveles de $\mathrm{FCH}$ y la clasificación de Child-Pugh; sin embargo, se observaron niveles más elevados en pacientes con enfermedad descompensada. Se determinó una asociación lineal positiva entre el FCH y la dureza hepática estimada por elastografía $\left(b=0,53 ; r^{2}=0,26 ; p=0,002\right.$ ) y una asociación lineal negativa con la albúmina $\left(b=-0,62 ; r^{2}=0,39 ; p<0,001\right)$. Únicamente la albúmina conservó esta asociación en el análisis multivariante. Conclusión: el FCH es un marcador de severidad en la cirrosis hepática. La albúmina y el grado de fibrosis determinada por ET se asociaron con niveles de FCH.
\end{abstract}

\section{Palabras clave}

Cirrosis hepática, factor de crecimiento hepático, elastografía transitoria, Child-Pugh-Turcotte.

\begin{abstract}
Introduction: Hepatocyte growth factor (HGF) is known to be a potent mitogenic agent both in vivo and in vitro. The role of HGF in cirrhosis is not completely clear, but some studies point to it as a marker of severity in cirrhosis, acute liver failure and chronic hepatitis. Objective: The objective of this study was to determine the relationship between HGF and the stage of hepatic cirrhosis and to identify factors associated with HGF levels in this population. Methodology: All patients with hepatic cirrhosis treated from January to March 2014 were evaluated. At the time patients were enrolled in the study their clinical histories were taken and they underwent transient elastography and extraction of samples for measurement of HGF. Results: No relationships were found between HGF levels and Child-Pugh classifications, however, higher levels of HGF were observed in patients with decompensated disease. A positive linear relations was found between HGF and hepatic hardness estimated by elastography $\left(b=0.53, r^{2}=0.26, p=0.002\right)$ and a negative linear relation was found between HGF and albumin $\left(b=-0.62, r^{2}=0.39, p<0.001\right)$. Only albumin retained this association in the multivariate analysis. Conclusion: HGF is a marker of severity in liver cirrhosis. Albumin and the degree of fibrosis determined by transient elastography were associated with HGF levels.
\end{abstract}

\section{Key words}

Hepatic cirrhosis, hebatocyte growth factor, transient elastography, Child-Turcotte-Pugh Classification. 


\section{INTRODUCCIÓN}

La cirrosis hepática es el estadio final de la actividad necroinflamatoria hepática mantenida de origen viral, metabólico o autoinmune. Hasta hace relativamente poco tiempo se consideraba que la cirrosis era una condición única y estática; sin embargo, actualmente se acepta que coexisten bajo este mismo diagnóstico subgrupos de pacientes con diferentes grados de lesión hepática $(1,2)$, con implicaciones pronósticas dentro de la historia natural de la enfermedad (3).

En el hígado, el factor de crecimiento hepático $(\mathrm{FCH})$ es conocido por ser un potente agente mitogénico tanto in vivo como in vitro (4-6) y un inductor de la regeneración hepática por estimular la síntesis del $\mathrm{ADN}$ en hepatocitos $(7,8)$. Varios investigadores han proporcionado evidencias directas de un papel esencial para la ruta $\mathrm{FCH} /$ Met durante la regeneración hepática, en modelos murinos de ratones knock-out condicionales de Met en el hígado (9-12).

Sin embargo, las implicaciones clínicas de los niveles de FCH en la cirrosis no están completamente claras y a pesar de tener un papel fundamental en los procesos de regeneración hepática (13), los estudios realizados lo señalan como un marcador de severidad en la cirrosis, en la insuficiencia hepática aguda $(14,15)$ y en la hepatitis crónica (14). Incluso se ha sugerido su papel como predictor de mortalidad en pacientes con insuficiencia hepática fulminante (IHF) (14).

La elastografía transitoria (ET) es una novedosa técnica que permite evaluar el grado fibrosis hepática de forma no invasiva $(16,17)$, y se ha convertido en una herramienta útil para estudiar a pacientes con cirrosis (18-21). Aunque se ha encontrado una asociación significativa entre el FCH y la presencia de fibrosis en la hepatitis crónica (14), esta relación nunca ha sido evaluada en sujetos con cirrosis. Dado que los valores de la ET se incrementan de la mano con el grado de fibrosis a lo largo de todo el espectro de severidad de la enfermedad hepática, incluida la cirrosis, es posible que pueda ser útil para evaluar esta asociación en este grupo de pacientes.

Los objetivos de este estudio fueron determinar la relación entre el FCH y el estadio de la cirrosis hepática e identificar los factores asociados con la los niveles de FCH en esta población.

\section{METODOLOGÍA}

Se evaluaron de forma sistemática todos los pacientes con diagnóstico inequívoco de cirrosis hepática por criterios clínicos, radiológicos o histológicos, valorado en la consulta externa de Soluciones Integrales en Gastroenterología y Hepatología, centro de referencia de la consulta de hepatología de primer nivel de cuatro de las más grandes EPS de la ciudad de Cartagena, desde enero hasta marzo de
2014. Todos los pacientes firmaron un consentimiento informado previo. La estimación de la dureza hepática a través de la ET y la recopilación de la información clínica y analítica fueron realizadas durante la misma mañana de la extracción de la muestra para la determinación del FCH. Los pacientes con diagnóstico de hepatocarcinoma o con antecedentes de hospitalización por descompensación de la enfermedad hepática durante los 30 días previos a la evaluación fueron excluidos del estudio.

\section{Estadificación de la enfermedad hepática}

\section{Clasificación de Child-Pugh-Turcotte}

La clasificación de Child-Pugh se utiliza para el estadiaje de la cirrosis. Se consideró que aquellos pacientes con un ChildPugh-Turcotte grado A se encontraban en la fase compensada y los que presentaban grado B-C, en la descompensada.

\section{Determinación del factor de crecimiento hepático}

Para la determinación en suero del factor de crecimiento hepático, se recolectó una muestra de sangre en tubos vacutainer esteriles (Becton-Dickimson Vacutainer System) y centrifugado a $3500 \mathrm{rpm}$ durante 15 minutos a $4{ }^{\circ} \mathrm{C}$. Para la cuantificación de $\mathrm{FCH}$, se utilizó el kit de ELISA (Cat $\mathrm{N}^{\circ} \mathrm{KAC} 2211$, Invitrogen) siguiendo las consideraciones del fabricante, basado en captura de antígeno y detección mediante inmunocomplejos por sándwich indirecto con límite de detección de $20 \mu \mathrm{g} / \mathrm{mL}$.

\section{Elastografía transitoria}

Para la estimación de la rigidez hepática se utilizó la ET mediante un fibroscan (Ecosense) serie 502. Este aparato emite una onda vibratoria que atraviesa el parénquima hepático. La velocidad a la cual recorre esta onda el órgano genera una estimación del grado de fibrosis que se expresa en kilopascales (Kpa): a mayor velocidad, mayor grado de fibrosis, y viceversa. Todos los pacientes guardaron ayuno durante al menos 8 horas. El procedimiento se efectuó en decúbito supino con el brazo derecho en abducción máxima. Las determinaciones fueron llevadas a cabo sobre la línea axilar media. Para la evaluación de la rigidez hepática se tomó en cuenta el valor mediano de 10 estimaciones; los resultados que incumplían los criterios de calidad del fabricamente no se tomaron en cuenta para el análisis. Un rango intercuartílico $<30 \%$ y una tasa de éxito (número de mediciones válidas/mediciones totales) $>60 \%$ se consideraron indispensables para evaluar la fiabilidad del resultado. Todos los pacientes se realizaron el procedimiento de forma gratuita como parte del protocolo del estudio para estimar la relación entre la elastografía y la calidad de vida y en ningún caso para confirmar el diagnóstico de cirrosis. 


\section{Análisis estadístico}

El análisis estadístico se realizó utilizando el programa PASW stastistics 15.0 (SPSS, Inc, Chicago). Todos los valores de $\mathrm{p}$ fueron calculados de forma bilateral. El nivel de significación estadística se fijó en $p<0,05$. Para describir las características de los pacientes se utilizó la mediana y los porcentajes para las variables cuantitativas y categóricas, respectivamente. Se emplearon las pruebas $t$ de student, ANOVA y de Ji-cuadrado para realizar las comparaciones entre grupos. Los factores asociados con los niveles de FCH se identificaron inicialmente a través de un análisis por regresión lineal univariante. Aquellas variables identificadas en esta fase se incluyeron en el análisis multivariante.

\section{RESULTADOS}

Finalmente 38 pacientes estuvieron disponibles para el análisis (figura 1): 28 con enfermedad compensada y 12 en la fase descompensada. La muestra estuvo constituida por una proporción similar de hombres y mujeres. Ninguno de los pacientes evaluados presentaba ascitis en el momento de la inclusión. Todas las estimaciones elastográficas incluidas en el análisis cumplieron con los criterios de calidad estipulados por el fabricante (rango intercuartílico $<30 \%$ del valor mediano; tasa de éxitos $>60 \%$ ). La media de edad, de la ET y del factor de crecimiento hepático fue de 61,5 años (DE 15,8), 29,6 Kpa (DE 18,5) y 916 pg/mL (DE 612 ), respectivamente. No se observaron diferencias significativas en el sexo, régimen de salud, variables antropométricas y niveles de transaminasas entre sujetos compensados y descompensados. Sin embargo, la etiología alcohólica $\mathrm{y}$ viral fue más frecuente en los pacientes compensados ( $\mathrm{p}$ $=0,003$ ). Las características de la población en función del estadio de la enfermedad se describen en la tabla 1.

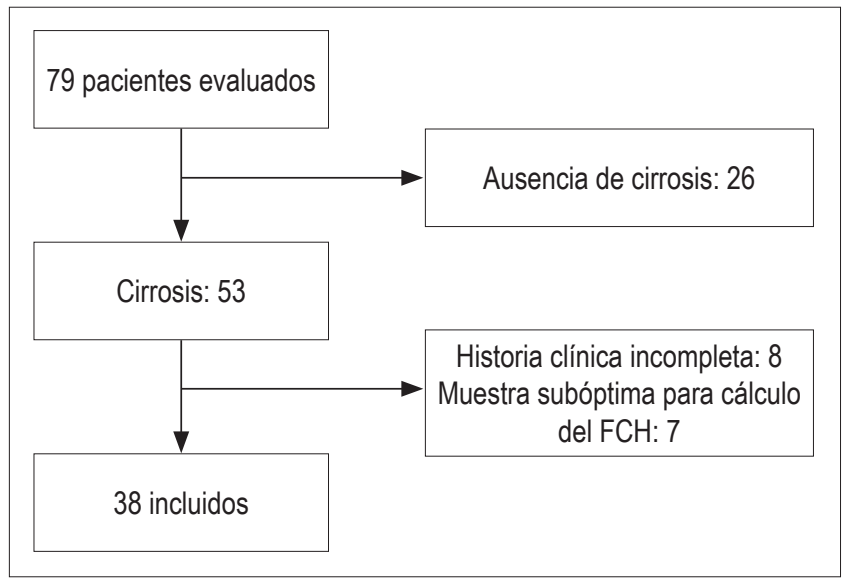

Figura 1. Diagrama de inclusión de los pacientes en el estudio.
Tabla 1. Variables sociodemográficas y clínicas en función del estadio de la enfermedad

\begin{tabular}{llll}
\hline \multicolumn{1}{c}{ Variables } & \multicolumn{1}{c}{$\begin{array}{c}\text { Compensado } \\
(\mathbf{n = 2 6 )}\end{array}$} & $\begin{array}{c}\text { Descompensado } \\
(\mathbf{n = 1 2})\end{array}$ & $\mathbf{p}$ \\
\hline Edad & $60(17,3)$ & $63(12,8)$ & 0,68 \\
Sexo & $12(46 \%)$ & $5(41 \%)$ & 0,79 \\
FCH & $867(641)$ & $1022(557)$ & 0,47 \\
Peso & $75(14,4)$ & $68(12,4)$ & 0,26 \\
Talla & $1,63(0,08)$ & $1,64(0,04)$ & 0,7 \\
Fibroscan & $28,5(20)$ & $31,9(14,9)$ & 0,63 \\
PLT & $166,966(144,149)$ & $130,037(74,422)$ & 0,43 \\
INR & $1,19(0,25)$ & $1,67(1,03)$ & 0,17 \\
BT & $0,91(0,45)$ & $2,6(1,96)$ & 0,01 \\
CR & $0,88(0,17)$ & $1,10(0,27)$ & 0,001 \\
Albúmina & $3,9(0,63)$ & $3,3(0,67)$ & 0,01 \\
GPT & $62,3(48)$ & $53,9(34)$ & 0,62 \\
GOT & $62,5(43)$ & $63,7(42)$ & 0,94 \\
Régimen & & & \\
Contributivo & $4(15)$ & $3(27)$ & 0,69 \\
Subsidiado & $17(65)$ & $6(54)$ & \\
Particular & $5(19)$ & $2(18)$ & \\
Várices & $11(44)$ & $8(72)$ & 0,23 \\
Etiología & & & \\
OH & $1(3,8)$ & $0(0)$ & 0,003 \\
VHC & $4(15,4)$ & $0(0)$ & \\
VHB & $5(19,2)$ & $2(16,7)$ & \\
NASH & $11(42.3)$ & $4(33,3)$ & \\
CRIPTOG & $0(0)$ & $6(50)$ & \\
CBP-AlH & $5(19.2)$ & $0(0)$ & \\
\hline
\end{tabular}

\section{Relación entre el factor de crecimiento hepático y estadio de la cirrosis hepática}

Los niveles más elevados de $\mathrm{FCH}$ se observaron en pacientes con enfermedad hepática descompensada (867 versus $1022 \mathrm{pg} / \mathrm{mL}$ ) (figura 2). Sin embargo, estas diferencias no alcanzaron la significación estadística $(\mathrm{p}=0,47)$. El análisis de otros factores relacionados con el estadio de la enfermedad encontró una asociación lineal positiva entre el FCH y la dureza hepática estimada por elastografía $\left(b=0,53 ; r^{2}=\right.$ $0,26 ; p=0,002)$ (figura 3 ), y una asociación lineal negativa con la albúmina $\left(b=-0,62 ; r^{2}=0,39 ; p<0,001\right)$ (figura 4). De esta manera, los niveles de FCH disminuyeron a medida que se incrementaron los de esta última. Esta relación se mantuvo en el análisis multivariante, en el que la albúmina fue el único predictor independiente de los niveles de $\mathrm{FCH}$ (tablas 2 y 3 ). 


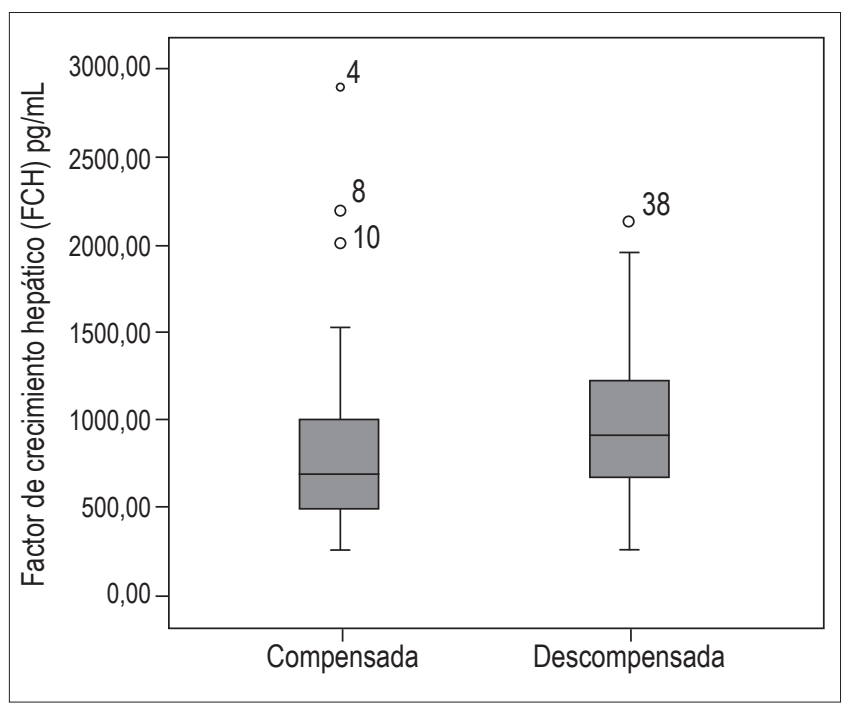

Figura 2. Diagrama de cajas de los niveles de FCH en función de la presencia de descompensación.

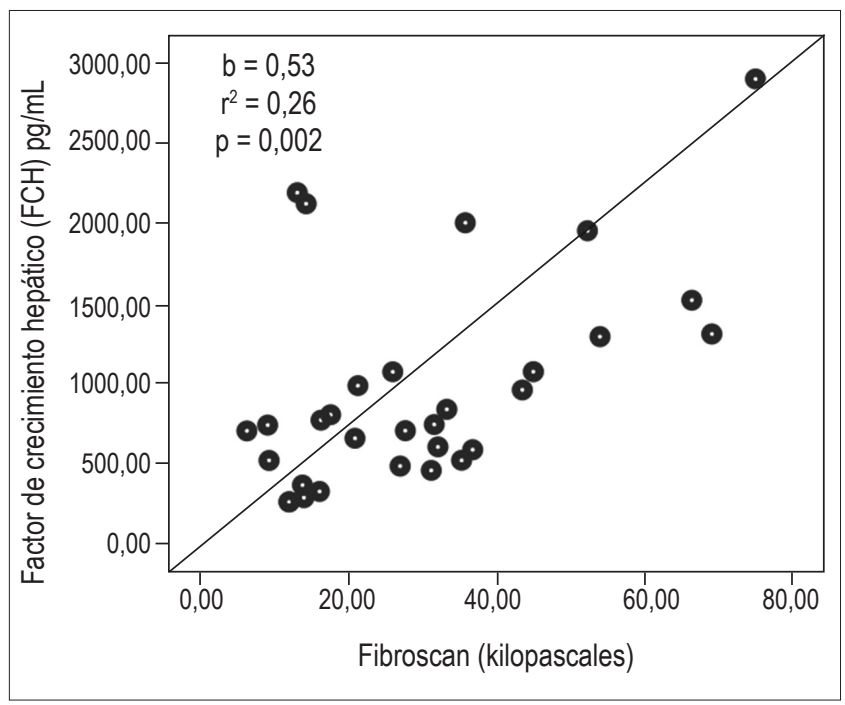

Figura 3. Relación entre la dureza hepática y el FCH. Análisis por regresión lineal univariante.

\section{DISCUSIÓN}

Como se ha descrito previamente, nuestros datos señalan una relación entre el FCH y el estadio de la cirrosis hepática $(14,15)$. La albúmina y la ET, ambos marcadores de severidad en la cirrosis $(3,19-21)$, tuvieron una relación lineal y significativa con los niveles plasmáticos de $\mathrm{FCH}$. Este último fue identificado inicialmente en el suero de ratas sometidas a una hepatectomía (4). Ulteriormente se demostró su importante papel en los procesos de regeneración hepática tanto in vivo como in vitro (22-24) al estimular la síntesis de $\mathrm{ADN}$ en los hepatocitos $(7,8)$.

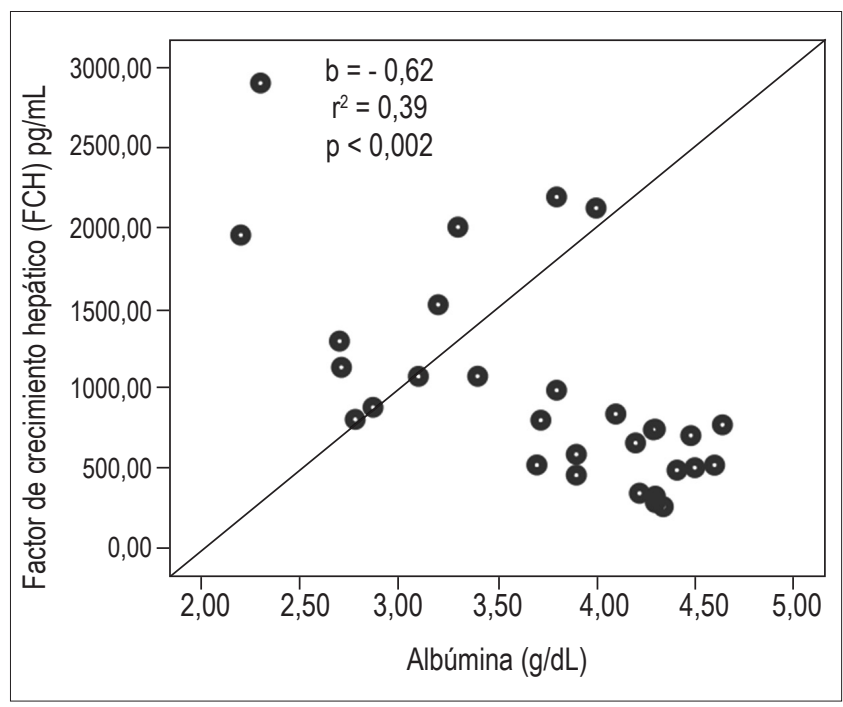

Figura 4. Relación entre la albúmina y el FCH. Análisis por regresión lineal univariante.

Tabla 2. Análisis de regresión lineal univariante. Variable dependiente: $\mathrm{FCH}(\mathrm{ng} / \mathrm{mL})$

\begin{tabular}{|c|c|c|c|}
\hline \multicolumn{4}{|c|}{ Regresión lineal univariante } \\
\hline Variable & B & IC $95 \%$ & $\mathrm{p}$ \\
\hline Fibroscan & 18,9 & $7,3-30,5$ & 0,002 \\
\hline Sexo & -270 & $(-681)-141$ & 0,19 \\
\hline Child puntos & 77,8 & $(-41.7)-197$ & 0,19 \\
\hline Várices & 134 & $(-595)-326,6$ & 0,55 \\
\hline PLT & $-0,001$ & $(-0,003)-0,001$ & 0,32 \\
\hline INR & 29,9 & $(-80)-139$ & 0,58 \\
\hline BT & 59 & $(-102)-221.9$ & 0,46 \\
\hline Albúmina & $-628,28$ & $(-907)-(-349)$ & $<0,001$ \\
\hline CR & $-315,5$ & $(-1428)-797,5$ & 0,56 \\
\hline GOT & 4,9 & $(-0,71)-10,6$ & 0,08 \\
\hline GPT & 0,94 & $0,32-0,74$ & 0,74 \\
\hline \multicolumn{4}{|c|}{ Coeficiente de correlación } \\
\hline Variable & & Pearson & $\mathrm{p}$ \\
\hline FIBROSCAN & & 0,53 & 0,01 \\
\hline PLT & & 0,18 & ns \\
\hline INR & & 0,1 & ns \\
\hline BT & & 0,11 & ns \\
\hline Albúmina & & $-0,62$ & 0,01 \\
\hline CR & & 0,09 & ns \\
\hline GOT & & 0,31 & ns \\
\hline GPT & & 0,06 & ns \\
\hline
\end{tabular}

BT: bilirrubina total; CR: creatinina; PLT: platelets; GOT: transaminasa glutámico-oxalacético; GPT: transaminasa glutámico-pirúvica; INR: índice internacional normalizado. 
Tabla 3. Análisis de regresión lineal multivariante. Variable dependiente: $\mathrm{FCH}(\mathrm{ng} / \mathrm{mL})$

\begin{tabular}{lccc}
\hline \multicolumn{4}{c}{ Regresión lineal multivariante FCH } \\
\hline \multicolumn{1}{c}{ Variable } & B & IC 95\% & p \\
\hline Albúmina & $-864,3$ & $(-1478)-(-249,8)$ & 0,008 \\
FIBROSCAN & $-6,5$ & $(-29,1)-(16,1)$ & 0,55 \\
\hline
\end{tabular}

En las enfermedades hepáticas, los niveles séricos de FCH pueden incrementarse debido a un aumento de la producción y una disminución del aclaramiento hepático, ya que el hígado es el órgano a través del cual se elimina la mayor parte del FCH de la circulación (25). Cuando los niveles de FCH se incrementan en la lesión hepática crónica, una de sus acciones fisiológicas es inducir la activación de las células progenitoras hepáticas, lo que favorece la restauración del tejido (26) a través del incremento de ciclinas y cinasas dependientes de ciclinas, que desempeñan un papel crítico en la progresión del ciclo celular en condiciones de daño hepático (8).

Así, en referencia a la asociación de la severidad de la enfermedad hepática con el $\mathrm{FCH}$, sugiere un efecto dual contrario de la vía $\mathrm{FCH} / \mathrm{cMet}$, a través de la que se estimula la regeneración. Es posible que en la lesión hepática crónica, el incremento del estrés oxidativo, de las especies reactivas de oxígeno generadas en la mitocondria de los hepatocitos y el nivel de lípidos peroxidados provenientes del tejido hepático necrosado mantengan en constante activación a las células hepáticas estrelladas, favoreciendo así la fibrogénesis en el tejido hepático (27). Este microambiente podría activar señales de inhibición del proceso regenerativo que lleven al bloqueo de la síntesis de $\mathrm{ADN}$ en el hepatocito, que estimula la producción de FCH y explica la elevación de los niveles plasmáticos a medida que se incrementa la severidad de la enfermedad.

Sin embargo, los efectos del FCH sobre el hígado cirrótico no se han establecido con claridad; son necesarios estudios que permitan identificar las vías de señalización que activa el FCH en la cirrosis y los mecanismos que evitan que se desarrolle un proceso de regeneración hepática eficiente.

La evidencia disponible señala al FCH como un marcador de severidad de la enfermedad hepática. En efecto, algunos estudios han encontrado una asociación positiva entre la clasificación de Child-Pugh-Turcotte, la bilirrubina, la albúmina, el tiempo de protrombina, AST y algunas citocinas proinflamatorias y el FCH $(14,15)$.

Incluso los niveles de $\mathrm{FCH}$ parecen tener implicaciones pronósticas, observándose una disminución de la supervivencia en pacientes con niveles elevados de FCH en el FHF (14). De igual manera se ha asociado con la progresión de la fibrosis y la necesidad de trasplante hepático en pacientes con atresia biliar que han recibido tratamiento quirúrgico (28) y con la aparición de carcinoma hepatocelular (29).

Recientemente se ha estudiado el comportamiento del FCH y la interleucina 6 (IL-6) en 60 pacientes con cirrosis de origen alcohólico (15). Los niveles de FCH fueron significativamente superiores en pacientes Child $\mathrm{B}$ y $\mathrm{C}$ en comparación con los Child A y en pacientes cirróticos frente al grupo control. Este estudio, que incluye el mayor número de pacientes cirróticos a la fecha, aporta una evidencia sólida sobre la relación entre el FCH y el estadio de la enfermedad.

Aunque los pacientes con enfermedad hepática descompensada en nuestro estudio tuvieron niveles superiores de $\mathrm{FCH}$, esta relación no pudo confirmarse $(\mathrm{p}=0,47)$. El tamaño de la muestra y el pequeño número de sujetos incluidos en la fase descompensada son probablemente la causa de estos resultados. Por otra parte, el grupo de pacientes incluidos en los otros estudios que estudiaron esta asociación presentaban una enfermedad más severa que nuestra muestra, con niveles de bilirrubina e INR más elevados y de albúmina más bajos. Esta circunstancia ha podido ocasionar que las diferencias de los niveles de $\mathrm{FCH}$ entre pacientes compensados y descompensados fueran más pronunciadas, lo que facilita la identificación de la relación observada entre el FCH y la clasificación de ChildPugh-Turcotte por estos autores $(14,15)$.

Por otra parte, la albúmina que hace parte de los componentes del Child-Pugh-Turcotte, que se utiliza de forma rutinaria para la evaluación de la capacidad de biosíntesis hepática y además ha demostrado ser un marcador pronóstico de progresión en pacientes con cirrosis compensada (3), estuvo estrechamente ligada a los niveles de $\mathrm{FCH}$, de tal manera que a medida que disminuye la albúmina se incrementan los niveles de FCH. Estos hallazgos son similares a los encontrados previamente y apoyan el papel del FCH como marcador de severidad en la enfermedad hepática crónica (14).

La cirrosis ha dejado de considerarse como un estadio único y estático de la enfermedad hepática crónica y actualmente se reconoce que existe un amplio espectro de pacientes, desde el punto de vista histológico, clínico y hemodinámico, dentro de los pacientes con cirrosis con un pronóstico diferente de progresión y de mortalidad $(1,30)$.

Un estudio que analizó la asociación entre la histología hepática y la presencia de hipertensión portal clínicamente significativa demostró que, en cuanto a la cirrosis, pueden existir diferentes grados de lesión histológica en pacientes clasificados en el mismo estadio de la enfermedad $(1,30)$.

La ET es capaz de determinar el grado de fibrosis hepática a través de la estimación de la dureza del tejido utilizando una onda vibratoria. Sus valores se incrementan de forma paralela al grado de lesión hepática (19-21). 
En pacientes con cirrosis establecida, la elastografía es capaz de identificar a una subpoblación de pacientes con un grado de lesión histológica más severa, con mayor riesgo de descompensación y de aparición de carcinoma hepatocelular (19-21). La relación lineal positiva entre la ET y el FCH observada en este estudio refleja su asociación con la severidad de la enfermedad hepática. Hallazgos similares han sido obtenidos con otros marcadores bioquímicos y clínicos característicos de las etapas más avanzadas de la cirrosis $(14,31)$.

Por otra parte, este estudio es el primero en evaluar la relación entre la ET y el FCH. Aunque estudios previos describieron una relación entre el grado de fibrosis y el FCH en pacientes con hepatitis crónica (14), esta relación no había sido evaluada previamente en pacientes con cirrosis establecida. La ET es una herramienta útil en este contexto para evitar el riesgo de sangrado y las otras complicaciones asociadas con la biopsia hepática, a la vez que ofrece una estimación precisa y objetiva del grado de lesión histológica que evita los sesgos de las escalas cualititativas utilizadas habitualmente para cuantificar el grado de fibrosis, incrementando así la reproducibilidad y la solidez de los resultados.

Por último, nuestros hallazgos apoyan el creciente cuerpo de información científica que reconoce que en la cirrosis coexisten diferentes grados de lesión histológica $(1,30)$ al establecer dentro de una muestra de pacientes con cirrosis una relación lineal entre la ET y el FCH similar a la observada con la albúmina, la bilirrubina y el tiempo de protrombina, todos ellos marcadores de severidad de la enfermedad hepática crónica $(14,15)$.

\section{CONCLUSIÓN}

El factor de crecimiento hepático $(\mathrm{FCH})$ es un marcador de severidad en la cirrosis hepática. La albúmina y el grado de fibrosis determinados por elastografía transitoria (ET) se asociaron con los niveles de FCH.

\section{Agradecimientos}

A la Universidad de Cartagena y Colciencias, por apoyar este proyecto de investigación a través del programa de Jóvenes Investigadores.

\section{REFERENCIAS}

1. Nagula S, Jain D, Groszmann RJ, et al. Histologicalhemodynamic correlation in cirrhosis-a histological classification of the severity of cirrhosis. J Hepatol. 2006;44:111-7.

2. Sethasine S, Jain D, Groszmann RJ, et al. Quantitative histological-hemodynamic correlations in cirrhosis. Hepatology. 2012;55:1146-53.

3. Ripoll C, Groszmann R, Garcia-Tsao G, et al. Hepatic venous pressure gradient predicts clinical decompensation in patients with compensated cirrhosis. Gastroenterology. 2007; 133:481-8.

4. Nakamura T, Nawa K, Ichihara A. Partial purification and characterization of hepatocyte growth factor from serum of hepatectomized rats. Biochem Biophys Res Commun. 1984;122:1450-9.

5. Russell WE, McGowan JA, Bucher NL. Partial characterization of a hepatocyte growth factor from rat platelets. J Cell Physiol. 1984;119:183-92.

6. Fabregat I, de Juan C, Nakamura T, et al. Growth stimulation of rat fetal hepatocytes in response to hepatocyte growth factor: modulation of c-myc and c-fos expression. Biochem Biophys Res Commun. 1992;189:684-90.

7. Michalopoulos GK, DeFrances MC. Liver regeneration. Science. 1997;276:60-6.

8. Fausto N. Liver regeneration. J Hepatol. 2000;32:19-31.

9. Huh CG, Factor VM, Sanchez A, et al. Hepatocyte growth factor/c-met signaling pathway is required for efficient liver regeneration and repair. Proc Natl Acad Sci. 2004; 101:4477-82.

10. Borowiak M, Garratt AN, Wustefeld T, et al. Met provides essential signals for liver regeneration. Proc Natl Acad Sci. 2004; 101:10608-13.

11. Phaneuf D, Moscioni AD, LeClair C, et al. Generation of a mouse expressing a conditional knockout of the hepatocyte growth factor gene: demonstration of impaired liver regeneration. DNA Cell Biol. 2004;23:592-603.

12. Suarez-Causado A, Caballero-Diaz D, Bertran E, et al. HGF/ c-Met signaling promotes liver progenitor cell migration and invasion by an epithelial-mesenchymal transition-independent, phosphatidyl inositol-3 kinase-dependent pathway in an in vitro model. Biochim Biophys Acta. 2015;1853:2453-63.

13. Fujimoto J, Kaneda Y. Reversing liver cirrhosis: impact of gene therapy for liver cirrhosis. Gene Ther. 1999;6:305-6.

14. Shiota G, Okano J, Kawasaki H, et al. Serum hepatocyte growth factor levels in liver diseases: clinical implications. Hepatology. 1995;21:106-12.

15. Prystupa A, Kicinski P, Sak J, et al. Proinflammatory cytokines (IL-1alpha, IL-6) and hepatocyte growth factor in patients with alcoholic liver cirrhosis. Gastroenterol Res Pract. 2015;2015:532615.

16. Pavlov CS, Casazza G, Nikolova D, et al. Transient elastography for diagnosis of stages of hepatic fibrosis and cirrhosis in people with alcoholic liver disease. Cochrane Database Syst Rev. 2015; 1:CD010542.

17. Sandrin L, Fourquet B, Hasquenoph JM, et al. Transient elastography: a new noninvasive method for assessment of hepatic fibrosis. Ultrasound Med Biol. 2003;29:1705-13.

18. Ciccarelli N, Fabbiani M, Grima P, et al. Liver fibrosis is associated with cognitive impairment in HIV-positive patients. J Int AIDS Soc. 2014;17:19722.

19. Kitson MT, Roberts SK, Colman JC, et al. Liver stiffness and the prediction of clinically significant portal hypertension and portal hypertensive complications. Scand J Gastroenterol. 2015;50:462-9.

20. Pang JX, Zimmer S, Niu S, et al. Liver stiffness by transient elastography predicts liver-related complications and 
mortality in patients with chronic liver disease. PLoS One. 2014;9:e95776.

21. Singh S, Fujii LL, Murad MH, et al. Liver stiffness is associated with risk of decompensation, liver cancer, and death in patients with chronic liver diseases: a systematic review and meta-analysis. Clin Gastroenterol Hepatol. 2013;11:1573-84.

22. Tajima H, Nakamura T. [Function, molecular structure and gene expression regulation of hepatocyte growth factor and its receptor]. Nihon Rinsho. 1992;50:1918-25.

23. Kinoshita T, Tashiro K, Nakamura T. Marked increase of HGF mRNA in non-parenchymal liver cells of rats treated with hepatotoxins. Biochem Biophys Res Commun. 1989; 165:1229-34.

24. Hamanoue M, Kawaida K, Takao S, et al. Rapid and marked induction of hepatocyte growth factor during liver regeneration after ischemic or crush injury. Hepatology. 1992;16:1485-92.

25. Porowski D, Wirkowska A, Hryniewiecka E, et al. Liver failure impairs the intrahepatic elimination of interleukin-6, tumor necrosis factor-alpha, hepatocyte growth factor, and transforming growth factor-beta. Biomed Res Int. 2015;2015:934065.
26. Vessey CJ, de la Hall PM. Hepatic stem cells: a review. Pathology. 2001;33:130-41.

27. Jiang JX, Venugopal S, Serizawa N, et al. Reduced nicotinamide adenine dinucleotide phosphate oxidase 2 plays a key role in stellate cell activation and liver fibrogenesis in vivo. Gastroenterology. 2010;139:1375-84.

28. Uchida $\mathrm{K}$, Inoue $\mathrm{M}$, Otake $\mathrm{K}$, et al. The significance of serum hepatocyte growth factor levels in planning follow-up of postoperative jaundice-free patients with biliary atresia. J Pediatr Surg. 2006;41:1657-62.

29. Shiota G, Rhoads DB, Wang TC, et al. Hepatocyte growth factor inhibits growth of hepatocellular carcinoma cells. Proc Natl Acad Sci. 1992;89:373-7.

30. Garcia-Tsao G, Friedman S, Iredale J, et al. Now there are many (stages) where before there was one: In search of a pathophysiological classification of cirrhosis. Hepatology. 2010;51:1445-9.

31. D’Amico G, Garcia-Tsao G, Pagliaro L. Natural history and prognostic indicators of survival in cirrhosis: a systematic review of 118 studies. J Hepatol. 2006;44:217-31. 